\title{
The role of automated cytometry in the new era of cancer immunotherapy (Review)
}

\author{
MARCO DANOVA $^{1}$, MARTINA TORCHIO ${ }^{1,5}$, GIUDITTA COMOLLI ${ }^{2}$, \\ ANDREA SBRANA $^{3}$, ANDREA ANTONUZZO ${ }^{3}$ and GIULIANO MAZZINI ${ }^{4}$ \\ ${ }^{1}$ Department of Internal Medicine and Medical Oncology, Vigevano Civic Hospital, ASST of Pavia, \\ I-27029 Vigevano; ${ }^{2}$ Department of Microbiology and Virology and Biotechnology Laboratories, \\ IRCCS San Matteo Foundation, I-27100 Pavia; ${ }^{3}$ Department of Medical Oncology 2, University Hospital of Pisa, \\ I-56126 Pisa; ${ }^{4}$ Institute of Molecular Genetics IGM-CNR, I-27100 Pavia, Italy
}

Received March 13, 2018; Accepted August 9, 2018

DOI: $10.3892 / \mathrm{mco} .2018 .1701$

\begin{abstract}
The introduction in the clinical practice of several new approaches to cancer immunotherapy has greatly increased the interest in analytical methodologies that can define the immunological profile of patients in the clinical setting. This requires huge effort to obtain reliable monitoring tools that could be used to improve the patient's clinical outcome. The clinical applications of flow cytometry (FCM) in oncology started with the measurement of DNA content for the evaluation of both ploidy and cell cycle profile as potential prognostic parameters in the majority of human solid cancer types. The availability of monoclonal antibodies widely broadened the spectrum of clinical applications of this technique, which rapidly became a fundamental tool for the diagnosis and prognosis of malignant hematological diseases. Among the emerging clinical applications of FCM, the study of minimal residual disease in hematological malignancies, the quantification of blood dendritic cells in various types of tumors, the study of metastatic spread in solid tumors throughout both the analysis of circulating endothelial progenitor cells and the identification and characterization of circulating tumor cells, all appear very promising. More recently, an advanced single cell analysis technique has been developed that combines the precision of mass spectrometry with the unique advantages of FCM. This approach, termed mass cytometry, utilizes antibodies conjugated to heavy metal ions for the analysis of cellular proteins by a mass spectrometer. It provides measurement of over 100 simultaneous cellular parameters in a single sample and has evolved from a promising technology to a high recognized platform for multi-dimensional single-cell
\end{abstract}

Correspondence to: Dr Martina Torchio, ${ }^{5}$ Present address: ENETS Centre of Excellence, IRCCS Cancer National Institute (INT) Milano, I-20133 Milano, Italy

E-mail: marco_danova@asst-pavia.it

Key words: flow cytometry, mass cytometry, clinical oncology, cancer immunotherapy analysis. Should a careful standardization of the analytical procedures be reached, both FCM and mass cytometry could effectively become ideal tools for the optimization of new immunotherapeutic approaches in cancer patients.

\section{Contents}

1. Introduction

2. Cancer immunotherapy today

3. Flow cytometry in clinical oncology

4. Role of automated cytometry in cancer immunotherapy

5. Conclusions

\section{Introduction}

Anticancer immunotherapy has represented a revolution in the field of cancer therapy and has become a valuable option for many patients $(1,2)$, but immune parameters that can measure antitumor immune response are still lacking $(3,4)$.

It is technically challenging to translate the principles of cancer immunotherapy into clinical practice; with the development of immune monitoring assays being particularly problematic. In this review paper we focus on automated cytometric techniques $(5,6)$.

In the 1960s, flow cytometry (FCM) was introduced as an analytical technique able to measure various characteristics of single cells in suspension following excitation with a light source $(7,8)$. In clinical oncology, it was firstly used for the study of DNA content for cell ploidy and proliferation activity determination (9). The discovery of monoclonal antibodies (moAbs) together with the introduction of new fluorescent dyes with narrow excitation and emission spectra, allowed for a wide application of this technology, especially in hematological oncology $(10,11)$. In recent years, the application of FCM for analysis of the so called 'rare events', such as the presence of residual leukemic blasts in the bone marrow after treatment, the blood dendritic cells or those cell types that correlated with a metastatic event, such as endothelial progenitor cells and circulating tumor cells, has become more and more promising (12-14). 
Recently, a new analytical approach, called mass cytometry, which combines the precision of mass spectrometry with the power of flow cytometric analysis, has been developed. The application of this technique is expected to answer a multitude of biological questions with a single sample and it will significantly enhance our ability to evaluate complex cellular systems with unique opportunities for biomarker development. However, at the moment the quantity and complexity of data obtained with this technology requires some analytical considerations $(15,16)$.

In this paper, we briefly review some of the current clinical applications of FCM in Oncology and we discussed the potential of both FCM and mass cytometry in the field of immuno-oncology.

\section{Cancer immunotherapy today}

Cancer immunotherapy is a treatment that enhances the patient's immune system to make it capable of killing tumor cells. After years of mixed results, with hopes dashed by failures in the field of immuno-oncology, the role of immunotherapy as an effective anticancer therapy has been established (17). The success of immunotherapy depends on the combination actions of host immune cells and tumor microenvironment, and because of the complexity of these mechanisms, a deeper knowledge of these aspects will contribute to make immunotherapy more effective.

Cancer immunotherapies are based upon several strategies that range from stimulating effector mechanisms to inhibiting suppressive ones. Strategies to prompt activation of effector immune cells include vaccination with tumor antigens or amplification of antigen presentation mechanisms to increase the ability of the patient's immune system to produce an immune response against cancer cells (18). Additional stimulatory strategies include adoptive cellular therapy (e.g., administering immunocompetent cells directly to patients), the administration of oncolytic viruses for the initiation of systemic antitumor immunity, and the use of Abs that enhance T cell activity (19). Strategies to stop immunosuppressive mechanisms include some types of chemotherapy, the use of Abs as a mean to switch off regulatory $\mathrm{T}$ cells, and the use of antibodies against immune-checkpoint molecules, such as CTLA-4 and programmed cell death protein 1 (PD-1)/PD-ligand 1 (PD-L1) (20). Several cancer immunotherapies of these classes have been recently approved and durable benefit with manageable toxicity can be achieved.

Ipilimumab, a fully human immunoglobulin G1 (IgG1) MoAb, was the first targeted, anti-CTLA-4, checkpoint inhibitor. Data obtained from the randomized study showed that Ipilimumab significantly increased the lifespan of the melanoma patients. Ipilimumab was the first therapy to demonstrate a survival benefit for patients with metastatic melanoma (21), and it was approved by the US Food and Drug Administration (FDA) for the treatment of advanced melanoma in 2010 (22). More impressive than the mean survival benefit was the effect of Ipilimumab on long-term survival: $20.8 \%$ of ipilimumab plus dacarbazine treated patients survived more than three years compared with $12.2 \%$ of patients receiving Dacarbazine only survived same time (23). The first clinical study of PD-1 blockade for solid tumors began in 2006, using a human MoAb against PD-1 (Nivolumab). It was initially tested in patients with previously untreated metastatic melanoma $\mathrm{p}$ without v-Raf murine sarcoma viral oncogene homolog B (BRAF) mutation: $72.9 \%$ patients in the Nivolumab-arm achieved a 1 year-overall survival rate, a much higher rate than the $42.1 \%$ registered in the Dacarbazine-arm (as well as a longer progression-free survival) (24). Data obtained from clinical trials conducted in non-small cell lung cancer (NSCLC) patients showed a cancer regression rate of about $6-17 \%$, a significant increase in survival rates (e.g., 1- and 2-year survival rates of 62 and $43 \%$, respectively for melanoma or 1- and 2-year survival rates of 42 and $23 \%$, respectively for lung cancer) (25), and a durable partial or complete response in a significant portion of treated cancer pts (26).

Pembrolizumab, another PD-1 blockade antibody, has been approved for NSCLC with over 50\% PD-L1 expression as a first line therapy (27). In a phase 3 trial conducted by Rek and Colleagues, 305 patients, with previously untreated advanced NSCLC with PD-L1 expression on at least $50 \%$ of tumor cells and no sensitizing mutation of the epidermal growth factor receptor gene or translocation of the anaplastic lymphoma kinase gene, were randomized to receive either Pembrolizumab (at a fixed dose of $200 \mathrm{mg}$ every 3 weeks) or the investigator's choice of platinum-based chemotherapy. The median progression-free survival time was 10.3 months in the Pembrolizumab group vs. 6.0 months in the chemotherapy group. The response rate was higher in the Pembrolizumab group than in the chemotherapy group (44.8 vs. $27.8 \%$ ), and the median duration of response was longer (not reached vs. 6.3 months) (28).

On the other side of PD-1/PD-L1 axis, Atezolizumab, a PD-L1 inhibitor, demonstrated significant activity in previously treated NSCLC patients, where it produced a median overall survival of 11.4 months vs. 9.5 months in Docetaxel-treated patients (29). Improved efficacy was observed with increasing PD-L1 expression: In fact, patients with the lowest PD-L1 did not appear to benefit from Atezolizumab treatment. Atezolizumab is being further assessed as first line treatment in PD-L1+ locally advanced or metastatic NSCLC pts, and vs. Docetaxel as second- or third-line treatment in locally advanced or metastatic NSCLC pts (30).

However, effective patient selection tools have not always accompanied this impressive clinical development of anti-PD-1/PD-L1 inhibitors $(31,32)$. The results of major clinical studies reveal how, despite the remarkable survival benefit obtained with checkpoint inhibition immunotherapy in certain populations, around 40-60\% of patients will not benefit from these therapies (33). Additionally, these treatments are costly and might have some associated toxicities. To date, no such pre-treatment biomarker has been validated to the point of inclusion in standard-of-care therapeutic decision-making, although insights have emerged from the identification of certain post-treatment immune responses that seem to correlate with clinical outcome $(34,35)$. Thus, it is imperative to identify valid biomarkers of response that help us in optimizing patient selection (36). Given the dynamic nature of the immune system and the multiple elements involved in the complex immune response against cancer, developing biomarkers for immunotherapy is more challenging than developing biomarkers for targeted therapy (37-39). 
Novel technologies are also needed to support, facilitate, and accelerate the clinical translation of these forms of immunotherapy (40). Specifically, the technological needs include: i) rapid characterization of the tumor and its immune microenvironment at the time of diagnosis; ii) prediction models of therapy outcome to guide treatment decisions for each patient, and iii) characterization of new tools for treatment.

\section{Flow cytometry in clinical oncology}

DNA ploidy and cell cycle analysis. The analysis of nuclear DNA content for the evaluation of both ploidy and cell cycle profile in the large majority of human cancers has been made through FCM for more than 25 years $(9,11,41)$. At the beginning, this type of analysis addressed hematological malignancies but, with the development of reliable tissue disaggregation and staining techniques, together with increasingly sophisticated multiparametric analyses supported by advanced acquisition systems and dedicated software for data elaboration and display, it was extended to almost all solid cancers. In recent years an impressive number of articles have been published on the potential clinical utility of the data derived from analysis of DNA content by FCM, with particular attention to the prognostic value of tumor ploidy and proliferative activity. Early investigations focused on the correlation of these biological characteristics with known prognostic factors, in particular clinical stage and histologic grade. Second-generation studies tried to correlate tumor ploidy and/or proliferative activity with the patient clinical course. From these studies aneuploidy showed important biological implications in many of the more common human malignancies, but it did not always correlate with classical clinical-pathological parameters or with prognosis (9). Alternatively, the critical level of sensitivity of FCM in the detection of the so called near-diploid/aneuploidy prevented from its routine application (42). Tumor proliferative activity (S-phase fraction) has been studied less extensively, but it might also be an outcome predictor in some tumor types. In addition, through ploidy and proliferative activity measurements, flow cytometric studies have focused on the possibility to provide a biological rationale to assist oncologists in selecting the treatment for some tumors, i.e., to predict the outcome of specific treatment regimens $(43,44)$. However, the peculiar technical capabilities of FCM did substantially fail in the above mentioned applications and showed a relatively little impact in clinical oncology. To partially explain this point it must be considered that, both ploidy and proliferative activity should be evaluated by examining tumor specimens from homogeneous patient series within specifically designed clinical trials and this has not always been the case. As a result, we see that the major limitation to an even more extensive clinical application of these data is coming from the sometimes strong lack of concordance between the various studies. If this can be sometimes ascribed to an inadequate design of prospective studies (i.e., insufficient patient number or short follow up), in many cases the discrepancies come from some technical aspects of FCM applications. A large number of studies have been carried out using formalin-fixed, paraffin-embedded tumor specimens that permitted the retrospective analysis of archival specimens. However, there are three potential limitations to the use of the archival material. Firstly, due to fixation-induced variations in flourochrome binding, external diploid standards cannot be utilized to determine which G0/1 peak represents the diploid subpopulation. Secondly, a problem with the use of paraffin-embedded material is that enzymatic digestion, required to produce single-cell suspensions, always results in a variable amount of subcellular debris, which is superimposed to the DNA histogram: if this debris fraction is not taken into account, overestimation of tumor S-phase fraction can occur. Thirdly, the resolution of DNA histograms obtained from paraffin-embedded specimens, as already mentioned, is usually lower than that obtained from fresh material and this increases the risk that aneuploidy tumors with near-diploid DNA content can be misclassified. In summary, despite early optimism, the clinical applications of DNA content analysis by FCM did not show a real impact on the diagnosis of neoplastic diseases or in the determination of prognosis of cancer patients.

Immunophenotypic analysis. One of the most important routine clinical applications of FCM derives from the identification and quantitation of cellular antigens by the use of flourochrome-labelled MoAbs (10). FCM has the ability to simultaneously evaluate the expression of several Ags, as well as the physical properties (size and cytoplasmic complexity) of individual cells, and to identify both normal and abnormal cell populations.

The identification and classification of cells by the presence of surface antigens began with the revolution of the discovery of $\mathrm{T}$ and $\mathrm{B}$ cells and has expanded to the analysis of other cell types i.e. monocytes, macrophages and immature myeloid cells. During the 1980s and 1990s FCM became an essential methodology for lineage characterization of immature precursor in hematological neoplasms and it also played an important role for the diagnosis and classification of lymphoid cell neoplasms. Abnormal maturation patterns in myeloid cells have also been used to refine the diagnosis of myelodysplastic syndromes. In the last ten years we have seen impressive advances in flow cytometric instrumentation that, together with the availability of wide range of Abs and fluorochromes, have improved our ability to identify different normal and aberrant cell populations $(45,46)$. Flow cytometry in the onco-hematological setting can be applied for determining origin and stage of differentiation of leukemias and lymphomas, detecting early recurrence of hematological malignancies (thus providing prognostic and therapeutic information for patients with lymphoid neoplasms), monitoring chemotherapeutic treatments, and for post-monitoring of bone marrow transplantation procedures. Hence, flow cytometric immunophenotyping has become an indispensable tool for the diagnosis, classification, staging, and therapy monitoring in onco-hematology $(47,48)$.

'Rare event' analysis. One of the major advantages of FCM comes from its ability to obtain information from single cells identified inside a heterogeneous cellular population. A cell is considered 'rare' when the number of that specific subpopulation represents $<0.001 \%$ of the entire population. For an analytical methodology o be adequate for this analysis inside a more prevalent population, it should overcome the sources of artifacts that become apparent only when a very large numbers 
of cells are acquired. Flow cytometry has allowed most of the studies of rare cell populations and the most recent machines allow the detection of cells that represent $0.0001 \%$ of the entire cell pool. Some examples of rare event problems that can be studied by FCM include the study of the minimal residual disease (MRD) in hematological malignancies, the detection and quantification of blood-circulating dendritic cell subsets, as well as of circulating endothelial cells and their progenitors and, more recently, of circulating tumor cells (12-14).

Minimal residual disease in onco-hematology. Flow cytometry has become an essential tool for the characterization of hematological cancers and several technological improvements have allowed the identification of very small tumor populations that may survive after induction therapy, and represent the MRD. The wide choice of Abs used in MRD detection has helped to give a precise definition that identifies abnormal populations throughout the treatment. Improvements in FCM hardware have allowed for high rate of data collection and the availability of new fluorochromes have allowed the rare population of interest to be isolated with sufficient precision. This latter point, together with the low background signal, have allowed the detection of residual tumor populations in a population of normal cells. As far as the count of such rare events is concerned, the distribution is governed by Poisson statistics, so that precision increases when a higher number of cells is collected. In several hematological malignancies, identification of populations at frequencies of $0.001 \%$ and lower has been observed (49). In the clinical setting, the quantitation of MRD by FCM has been shown to correlate with the relapse and survival rates in some hematological diseases; for this reason, evidence of MRD is sometimes taken as a parameter to change therapy during treatment protocols (50).

Circulating dendritic cells. Dendritic cells (DC) are specialized phagocytes that possess a unique ability to sense perturbations in the tissue microenvironment and activate adaptive immune responses, whilst maintaining immunological tolerance. They are crucial antigen-presenting cells and play a primary role in antitumor immune response by controlling the initiation of the $\mathrm{T}$ cell-dependent immune response. Two peripheral blood DC subsets have been identified on the basis of their CD11c expression: The CD11c-negative (CD11c-) DCs (that express high levels of CD123), lymphoid-derived DCs, and the CD11c+/CD123-cells, myeloid-derived ones. In the last several years, several FCM studies have focused on the determination of the number and of the functional alterations of DCs and their subsets in different type of human cancers (51). Some of these studies have been conducted to investigate the contribution of blood-derived DCs to the mechanisms of the immune responses of patients with advanced cancer; this might help in the selection of potential candidates for active immunotherapy trials (48).

Circulating endothelial cells and endothelial progenitors. Circulating endothelial cells (CECs) represent a promising tool for the monitoring of the treatment response and of the clinical outcome in oncological patients, whereas endothelial progenitor cells (EPCs) are a blood-circulating cell population that is able to form endothelial colonies in vitro and to promote vasculogenesis (52). Both these cells have been proposed as non-invasive surrogate biomarkers of cancer angiogenesis. Their baseline number and kinetics in cancer patients have been widely studied and several previous experiences have demonstrated that they can be affected by both disease status and anti-cancer treatments $(53,54)$. Multiparameter FCM has been commonly used to quantify CECs and EPCs and their subpopulations, but this is still technically challenging, because at the moment there are no standardized protocols for their identification and counting. Major limitations include: i) Multiple flow cytometric protocols and methods; ii) heterogeneity in sample processing phases; iii) absence of consensus on standardized and validated MoAb panels for CECs and EPCs; iv) specific compensation and gating strategies; v) application of specific data analysis programs. The lack of a consensus on a valid CEC and EPC phenotypic definition and the multitude of the used flow cytometric methods have resulted in a great heterogeneity in the reported blood levels of CECs and EPCs, which limits the impact of the obtained data in clinical practice (53).

Circulating tumor cells. In the last 10 years, circulating tumor cells (CTCs) have been widely studied as new biomarkers, because they provide a non-invasive source of tumor material that can be evaluated throughout all the stages of disease management (including monitoring the response to therapy, identifying possible therapeutic targets, and assessing the development of drug resistance) (55-57). Blood-isolated cells can be used for phenotypic analysis, tumor genotyping, transcriptional profiling, as well as for ex vivo cell cultures (58). Prognostic value of CTC enumeration has been shown in several types of epithelial tumors and a worse survival has been observed in patients with a higher number of CTCs in breast, prostate, and colorectal cancer. Recent findings about CTCs suggest that the mere enumeration at a certain moment may not be sufficient as a prognostic and predictive biomarker. In particular, it is now known that CTCs are actually a heterogeneous population and the genetic and phenotypic characteristics of tumor cells can change over time, especially in those patients who undergo active treatments. Therefore, not only should CTCs be counted, but they should also be characterized at different moments of the care pathways. Moreover, CTC characterization could provide fundamental information about genotypic and phenotypic features of a tumor, thus sparing the patient the stress of an invasive procedure. There are a variety of novel technologies that are currently under study and development for the detection and analysis of rare circulating cells in cancer patients (56).

Flow cytometry was one of the first techniques used for the detection of blood CTCs (55). It is an excellent tool for this purpose, because it possesses the ability to easily recover viable cells with high purity that are suitable for downstream molecular analysis. In recent years, different automated cytometry techniques have been employed to further subdivide CTCs according to tumor origin and proliferative/invasive capacity, and FCM remains promising in this field. However, as a general consideration, although CTCs are already used in clinical trials as a possible biomarker, their routine clinical utility is still under active investigation. 


\section{Role of automated cytometry in cancer immunotherapy}

A wide variety of automated cytometry technologies are being explored to be clinically implemented as a possible help in the management of new immunotherapeutic strategies in clinical oncology. In fact, sensitive and accurate assays that determine the presence of cancer biomarkers and host immune responses may allow clinicians to more effectively determine the therapeutic response and allow better treatment strategies Other reliable technologies detect the presence of micrometastases and will allow routine and frequent testing after treatment and during remission or focus on the features of the tumor microenvironment that possibly hinder immunotherapy (58).

These technological assays do represent important challenges for bioengineers that hopefully may soon help translate the promise of immunotherapy into reality. This could be realized by developing the needed assays for effective patient monitoring.

Clinical cytometry has evolved from a technique that was primarily used to characterize large populations of abnormal cells to one that can routinely evaluate small populations of cells with abnormalities in antigen expression. These advances have expanded in recent years and the technique has become refined enough to be routinely applied to immunophenotyping.

Flow cytometry traditionally uses fluorochrome-labelled probes to identify cells expressing the target of those probes. A new platform for FCM has been developed and this includes the precision of mass spectrometry. The fusion of the two technologies, termed mass cytometry (cytometry by time-of-flight, CyTOF), provides the simultaneous measurement of over 40 parameters at a single-cell level, significantly increasing the possibility to evaluate complex cellular systems. In mass cytometry, the fluorescent probes are replaced by heavy metal isotopes that are chelated to a polymer, covalently linked to antibodies. After staining with these probes, cell suspension is introduced via an aereosol stream onto a plasma column, resulting in an ionization of the labelled cells. The heavy ions (derived by each single cell) are then focused into a time-of-flight detector $(15,59)$.

Both conventional FCM and mass cytometry allow the quantitation of multiple parameters in lots of individual cells by combining different phenotypic and functional markers and have the potential to give information on immune responsiveness in cancer patients. In particular, they are suitable to study the functional heterogeneity of cancer cells and to define predictive biomarkers for immunotherapy.

However, there are some technico-metodological warnings that need taking into account. In both analytical platforms, FCM and mass cytometry, there can be loss of cells in sample preparation and washing steps, although mass cytometry generally requires more washing steps than fluorescence assays. Collection speed is also much lower in mass cytometry (300-500 events per second, compared to several thousand event/second in fluorescence cytometry). Cells for these assays need to be in single-cell suspensions and debris and cell aggregates can interfere with the interpretation of data. Because of the potential cell loss, samples with $<10^{5}$ cells are usually not appropriate for either FCM or mass cytometry. Finally, both assays require good viable cells and this can be compromised by overnight shipping of blood.

\section{Conclusions}

Immune monitoring has become an essential tool in the field of new cancer immunotherapy and to clarify the mechanisms at the basis of a successful treatment. Flow cytometry is the typical multi-parameter analytical assay for the characterization of single cells in solution, and it has been largely utilized in preclinical immunology as well as in cancer immunotherapy trials. Applications of FCM in cancer immune monitoring include the characterization of tumor-antigen specificity of a patient's $\mathrm{T}$ cells by peptide-MHC multimers, the intracellular staining for effector cytokines, the evaluation of cytotoxicity, the measurement of the proliferation and the assessment of immune cells.

Mass cytometry is a relatively novel technology similar to FCM that has emerged as a competitive technical approach for multi-dimensional, single cell analysis. The use of probes that are labelled with heavy metal ions rather than fluorescence probes and then detected with time-of-flight mass spectrometry may allow the simultaneous detection of up to 40 parameters. The application of both techniques in the field of cancer immunotherapy is very promising and a number of related applications are under development. If implemented, this may allow for the generation of large amounts of multidimensional data that are amenable to high through-put analysis and this may offer unique opportunities and challenges for the field of biomarker development. The use of these powerful techniques will answer a multitude of questions with just one sample being analyzed and this will likely help to tailor cancer immunotherapies to each patient.

Given the technical complexity of both assays, reproducibility still represents a problem and this strongly underlines the need for a standardization at various levels, such as sample handling procedures, instrument set up, gating and analysis strategies and data reporting systems. Overcoming these challenges will make this technique perfect to optimize cancer immunotherapy, since we may learn more from clinical trials and we will be able to better predict how each individual patient should be treated. This will turn immunotherapy into a fundamental therapeutic strategy for a larger number of patients.

\section{Acknowledgements}

Not applicable.

\section{Funding}

No funding was received.

\section{Availability of data and materials}

Materials included in the manuscript, including all relevant raw data, will be made freely available to any researchers who wish to use them for non-commercial purposes, while preserving any necessary confidentiality and anonymity.

\section{Authors' contributions}

MD, MT, GC, AS, AA and GM contributed to study design and writing of the manuscript. 


\section{Ethics approval and consent for publication}

Not applicable.

\section{Patient consent for publication}

Not applicable.

\section{Competing interests}

The authors have no competing interests to declare.

\section{References}

1. Decker WK, da lva RF, Sanabria MH, Angelo LS, Guimarães F, Burt BM, Kheradmand F and Paust S: Cancer immunotherapy: Historical perspective of a clinical revolution and emerging preclinical animal models. Front Immunol 8: 829, 2017.

2. Farkona S, Diamandis EP and Blasutig IM: Cancer immunotherapy: The beginning of the end of cancer? BMC Med 14: 73, 2016.

3. Yuan J, Hegde PS, Clynes R, Foukas PG, Harari A, Kleen TO, Kvistborg P, Maccalli C, Maecker HT, Page DB, et al: Novel technologies and emerging biomarkers for personalized cancer immunotherapy. J Immunother Cancer 4: 3, 2016.

4. Butterfield LH: The society for immunotherapy of cancer biomarkers task force recommendations review. Semin Cancer Biol: Sept 22, 2017 (Epub ahead of print).

5. Maecker HT and Harari A: Immune monitoring technology primer: Flow and mass cytometry. J Immunother Cancer 3: 44, 2015.

6. Greenplate AR, Johnson DB, Ferrell PB Jr and Irish JM: Systems immune monitoring in cancer therapy. Eur J Cancer 61: 77-84, 2016.

7. Robinson JP and Roederer M: History of science. Flow cytometry strikes gold. Science 350: 739-740, 2015.

8. Blow N: Going with the flow. Biotechniques 62: 201-205, 2017.

9. Mazzini G and Danova M: Fluorochromes for DNA staining and quantitation. Methods Mol Biol 1560: 239-259, 2017 (Springer Science Business Media LLC).

10. Craig FE and Foon KA: Flow cytometric immunophenotyping for hematologic neoplasms. Blood 111: 3941-3967, 2008.

11. Adan A, Alizada G, Kiraz Y, Baran Y and Nalbant A: Flow cytometry: Basic principles and applications. Crit Rev Biotechnol 37: 163-176, 2017.

12. Irish JM and Doxie DB: High-dimensional single-cell cancer biology. Curr Top Microbiol Immunol 377: 1-21, 2014

13. Proserpio V and Lönnberg T: Single-cell technologies are revolutionizing the approach to rare cells. Immunol Cell Biol 94: 225-229, 2016.

14. Liang SB and Fu LW: Application of single-cell technology in cancer research. Biotechnol Adv 35: 443-449, 2017.

15. Spitzer MH and Nolan GP: Mass cytometry: Single cells, many features. Cell 165: 780-791, 2016.

16. Behbehani GK: Applications of mass cytometry in clinical medicine: The promises and perils of clinical CyTOF. Clin Lab Med 37: 945-964, 2017.

17. Chen DS and Mellman I: Elements of cancer immunity and the cancer-immune set point. Nature 541: 321-330, 2017.

18. Ozverel CS, Karaboz I and Nalbantsoy A: Novel treatment strategies in cancer immunotherapy. Acta Biol Turc 30: 36-51, 2017.

19. Eggermont LJ, Paulis LE, Tel J and Figdor CG: Towards efficient cancer immunotherapy: Advances in developing artificial antigen-presenting cells. Trends Biotechnol 32: 456-465, 2014.

20. Śledzińska A, Menger L, Bergerhoff K, Peggs KS and Quezada SA: Negative immune checkpoints on T lymphocytes and their relevance to cancer immunotherapy. Mol Oncol 9: 1936-1965, 2015.

21. Hodi FS, O'Day SJ, McDermott DF, Weber RW, Sosman JA, Haanen JB, Gonzalez R, Robert C, Schadendorf D, Hassel JC, et al: Improved survival with ipilimumab in patients with metastatic melanoma. N Engl J Med 363: 711-723, 2010

22. O'Day SJ, Maio M, Chiarion-Sileni V, Gajewski TF, Pehamberger H, Bondarenko IN, Queirolo P, Lundgren L, Mikhailov S, Roman L, et al: Efficacy and safety of ipilimumab monotherapy in patients with pretreated advanced melanoma: A multicenter single-arm phase II study. Ann Oncol 21: 1712-1717, 2010.
23. Robert C, Thomas L, Bondarenko I, O'Day S, Weber J, Garbe C, Lebbe C, Baurain JF, Testori A, Grob JJ, et al: Ipilimumab plus dacarbazine for previously untreated metastatic melanoma. $\mathrm{N}$ Engl J Med 364: 2517-2526, 2011.

24. Robert C, Long GV, Brady B, Dutriaux C, Maio M, Mortier L, Hassel JC, Rutkowski P, McNeil C, Kalinka-Warzocha E, et al: Nivolumab in previously untreated melanoma without BRAF mutation. N Engl J Med 372: 320-330, 2015.

25. Brahmer J, Reckamp KL, Baas P, Crinò L, Eberhardt WE, Poddubskaya E, Antonia S, Pluzanski A, Vokes EE, Holgado E, et al: Nivolumab versus docetaxel in advanced squamous cell-non-small cell lung cancer. N Engl J Med 373: $123-135,2015$.

26. Gettinger SN, Horn L, Gandhi L, Spigel DR, Antonia SJ, Rizvi NA, Powderly JD, Heist RS, Carvajal RD, Jackman DM, et al: Overall Survival and Long-Term Safety of Nivolumab (Anti-Programmed Death 1 Antibody, BMS-936558, ONO-4538) in patients with previously treated advanced nons-mall-cell lung cancer. J Clin Oncol 33: 2004-2012, 2015.

27. Garon EB, Rizvi NA, Hui R, Leighl N, Balmanoukian AS, Eder JP, Patnaik A, Aggarwal C, Gubens M, Horn L, et al: Pembrolizumab for the treatment of non-small-cell lung cancer. N Engl J Med 372: 2018-2028, 2015.

28. Reck M, Rodríguez-Abreu D, Robinson AG, Hui R, Csőszi T, Fülöp A, Gottfried M, Peled N, Tafreshi A, Cuffe S, et al: Pembrolizumab versus chemotherapy for PD-L1 positive non-small-cell lung cancer. N Engl J Med 375: 1823-1833, 2016.

29. Fehrenbacher L, Spira A, Ballinger M, Kowanetz M, Vansteenkiste J, Mazieres J, Park K, Smith D, Artal-Cortes A, Lewanski C, et al: Atezolizumab versus docetaxel for patients with previously treated non-small-cell lung cancer (POPLAR): A multicentre, open-label, phase 2 randomised controlled trial. Lancet 387: 1837-1846, 2016.

30. Seetharamu N, Preeshagul IR and Sullivan KM: New PD-L1 inhibitors in non-small cell lung cancer-impact of atezolizumab. Lung Cancer (Auckl) 8: 67-78, 2017.

31. Gridelli C, Ardizzoni A, Barberis M, Cappuzzo F, Casaluce F, Danesi R, Troncone G and De Marinis F: Predictive biomarkers of immunotherapy for non-small cell lung cancer: Results from an experts panel meeting of the italian association of thoracic oncology. Transl Lung Cancer Res 6: 373-386, 2017.

32. Liu D, Wang S and Bindeman W: Clinical applications of PD-L1 bioassays for cancer immunotherapy. J Hematol Oncol 10: 110, 2017.

33. Maleki Vareki S, Garrigós C and Duran I: Biomarkers of response to PD-1/PD-L1 inhibition. Crit Rev Oncol Hematol 116: 116-124, 2017.

34. Roussel H, De Guillebon E, Biard L, Mandavit M, Gibault L, Fabre E, Antoine M, Hofman P, Beau-Faller M, Blons $\mathrm{H}$, et al: Composite biomarkers defined by multiparametric immunofluorescence analysis identify ALK-positive adenocarcinoma as a potential target for immunotherapy. OncoImmunology 6: e1286437, 2017.

35. Quandt D, Dieter Zucht H, Amann A, Wulf-Goldenberg A, Borrebaeck C, Cannarile M, Lambrechts D, Oberacher H, Garrett J, Nayak T, et al: Implementing liquid biopsies into clinical decision making for cancer immunotherapy. Oncotarget 8: 48507-48520, 2017.

36. Milano G: Resistance to immunotherapy: Clouds in a bright sky. Invest New Drugs 35: 649-654, 2017.

37. Martens A, Wistuba-Hamprecht K, Geukes Foppen M, Yuan J, Postow MA, Wong P, Romano E, Khammari A, Dreno B, Capone M, et al: Baseline peripheral blood biomarkers associated with clinical outcome of advanced melanoma patients treated with Ipilimumab. Clin Cancer Res 22: 2908-2918, 2016.

38. Hegde PS, Karanikas V and Evers S: The where, the when, and the how of immune monitoring for cancer immunotherapies in the era of checkpoint inhibition. Clin Cancer Res 22: 1865-1874, 2016.

39. Masucci GV, Cesano A, Hawtin R, Janetzki S, Zhang J, Kirsch I, Dobbin KK, Alvarez J, Robbins PB, Selvan SR, et al: Validation of biomarkers to predict response to immunotherapy in cancer: Volume I-pre-analytical and analytical validation. J Immunother Cancer 4: 76, 2016

40. Krieg C, Nowicka M, Guglietta S, Schindler S, Hartmann FJ, Weber LM, Dummer R, Robinson MD, Levesque MP and Becher B: High-dimensional single-cell analysis predicts response to anti-PD-1 immunotherapy Nat Med 24: 144-153, 2018. 
41. Danielsen HE, Pradhan M and Novelli M: Revisiting tumor aneuploidy-the place of ploidy assessment in the molecular era. Nat Rev Clin Oncol 13: 291-304, 2016.

42. Mishra S, Awasthi NP, Husain N, Anand A, Pradeep Y, Ansari R and Saxena S: Flow cytometric analysis of DNA ploidy in liquid based cytology for cervical pre-cancer and cancer. Asian Pac J Cancer Prev 18: 1595-1601, 2017.

43. Pinto AE, Pereira T, Silva GL and André S: Prognostic relevance of DNA flow cytometry in breast cancer revisited: The 25-year experience of the portuguese institute of oncology of lisbon. Oncol Lett 13: 2027-2033, 2017.

44. Malcovati L, Hellström-Lindberg E, Bowen D, Adès L, Cermak J, Del Cañizo C, Della Porta MG, Fenaux P, Gattermann N, Germing U, et al: Diagnosis and treatment of primary myelodysplastic syndromes in adults: Recommendations from the European LeukemiaNet. Blood 122: 2943-2964, 2013.

45. Finak G, Langweiler M, Jaimes M, Malek M, Taghiar J, Korin S, Raddassi K, Devine L, Obermoser G, Pekalski ML, et al: Standardizing flow cytoanalysis from the human immunophenotyping consortium. Sci Rep 6: 20686, 2016.

46. Strati P and Shanafelt TD: Monoclonal B-cell lymphocytosis and early-stage chronic lymphocytic leukemia: Diagnosis, natural history, and risk stratification. Blood 126: 454-462, 2015.

47. Tognarelli S, Jacobs B, Staiger N and Ullrich E: Flow cytometry-based assay for the monitoring of NK cell functions. J Vis Exp: Oct 30, 2016. doi: 10.3791/54615.

48. Flores-Montero J, Sanoja-Flores L, Paiva B, Puig N, García-Sánchez O, Böttcher S, van der Velden VHJ, Pérez-Morán JJ, Vidriales MB, García-Sanz R, et al: Next generation flow for highly sensitive and standardized detection of minimal residual disease in multiple myeloma. Leukemia 31: 2094-2103, 2017

49. Luskin MR and Stone RM: Can minimal residual disease determination in acute myeloid leukemia be used in clinical practice? J Oncol Pract 13: 471-480, 2017.

50. Manzoni M, Rovati B, Ronzoni M, Loupakis F, Mariucci S, Ricci V, Gattoni E, Salvatore L, Tinelli C, Villa E and Danova M: Immunological effects of bevacizumab-based treatment in metastatic colorectal cancer. Oncology 79: 187-196, 2010.
51. Manzoni M, Mariucci S, Delfanti S, Rovati B, Ronzoni M, Loupakis F, Brugnatelli S, Tinelli C, Villa E, Falcone A and Danova M: Circulating endothelial cells and their apoptotic fraction are mutually independent predictive biomarkers in Bevacizumab-based treatment for advanced colorectal cancer. J Cancer Res Clin Oncol 138: 1187-1196, 2012.

52. Danova M, Comolli G, Manzoni M, Torchio M and Mazzini G: Flow cytometric analysis of circulating endothelial cells and endothelial progenitors for clinical purposes in oncology: A critical evaluation. Mol Clin Oncol 4: 909-917, 2016.

53. De Biasi S, Gibellini L, Feletti A, Pavesi G, Bianchini E, Lo Tartaro D, Pecorini S, De Gaetano A, Pullano R, Boraldi F, et al: High speed flow cytometry allows the detection of circulating endothelial cells in hemangioblastoma patients. Methods 134-135: 3-10, 2018.

54. Danova M, Torchio M and Mazzini G: Isolation of rare circulating tumor cells in cancer patients: Technical aspects and clinical implications. Expert Rev Mol Diagn 11: 473-485, 2011.

55. Kowalik A, Kowalewska M and Góźdź S: Current approaches for avoiding the limitations of circulating tumor cells detection methods-implications for diagnosis and treatment of patients with solid tumors. Transl Res 185: 58-84.e15, 2017.

56. Wu CP, Wu P, Zhao HF, Liu WL and Li WP: Clinical applications of and challenges in single-cell analysis of circulating tumor cells. DNA Cell Biol 37: 78-89, 2018.

57. Chen L, Bode AM and Dong Z: Circulating tumor cells: Moving biological insights into detection. Theranostics 7: 2606-2619, 2017.

58. D'Errico G, Machado HL and Sainz B Jr: A current perspective on cancer immune therapy: Step-by-step approach to constructing the magic bullet. Clin Transl Med 6: 3, 2017.

59. Tanner SD, Baranov VI, Ornatsky OI, Bandura DR and George TC: An introduction to mass cytometry: Fundamentals and applications. Cancer Immunol Immunother 62: 955-965, 2013. 